\title{
Acne in hirsute women
}

\author{
Besa Gacaferri Lumezi ${ }^{1}$, Hatixhe Latifi Pupovci ${ }^{1}$, Violeta Lokaj Berisha ${ }^{1}$, Aferdita Uka Goçi², Antigona Gerqari ${ }^{3}$
}

${ }^{1}$ Department of Physiology and Immunology, Medical Faculty, University of Prishtina, Kosovo ${ }^{2}$ Child and Adolescent Mental Health Center, University Clinical Center of Kosovo, Kosovo ${ }^{3}$ Department of Dermatology, Medical Faculty, University of Prishtina, Kosovo

Postep Derm Alergol 2014; XXXI, 6: 356-361 DOI: $10.5114 /$ pdia.2014.47118

\begin{abstract}
Introduction: Acne and hirsutism are common manifestations of hyperandrogenism.

Aim: To investigate whether or not acne is present in women with hirsutism, associated with different clinical, endocrine and ultrasonographic features.

Material and methods: The prospective study included 135 women with hirsutism, aged 14-46 years. We measured the levels of hormones with radioimmunoassay/immunoradiometric assay methods.

Results: Acne were present in 63 (47.6\%) women with hirsutism. Sixty women had mild forms of acne, including: whiteheads, blackheads, papules and pustules. Only 3 women had moderate to severe acne, including nodules. In a group of women with hirsutism and acne, 6 (9.5\%) were obese. In our study we found a high prevalence of androgen excess among hirsute women with acne: total testosterone was increased in $79 \%$, free testosterone in $20.6 \%$, androstenedione in 69.8\%, dehydroepiandrosterone sulfate (DHEAS) in 30.1\%, 17-OH-progesterone 68.2\% and sex hormone-binding globulin (SHBG) was decreased in $33.3 \%$ of women. Women with hirsutism and acne have received oral contraceptives for a year, without or in a combination with other medication. Thirty-four (53.9\%) women have shown improvement in hirsutism and acne.

Conclusions: In this study we found a high prevalence of acne in hirsute women. The prevalence of acne was higher in polycystic ovarian syndrome. Since these women have associated endocrine changes it is important to correct them with hormonal therapy.
\end{abstract}

Key words: acne, hirsutism, androgens, oral contraceptives.

\section{Introduction}

Acne is one of the most prevalent skin diseases. It is an infectious, inflammatory disorder of the sebaceous glands with local excess sebum production [1]. Androgens are in volved in the development of acne [2]. Increased androgen levels (testosterone, androstenedione, dehydroepiandrosterone, dehydroepiandrosterone sulfate) in women with acne have been repeatedly demonstrated in many studies. Androgens enhance the sebum production and follicular keratosis that plays the key role in the etiology of acne [3].

Disease states associated with hyperandrogenism, such as polycystic ovarian syndrome or androgen-secreting tumors, may be associated with acne vulgaris [4]. Acne and hirsutism are common manifestations of hyperandrogenism. Nevertheless, acne or hirsutism may be found with normal androgenic parameters. Increased sensitivity of sebaceous end organ to androgens and increased peripheral metabolism of androgen are other possible mechanisms involved in the development of acne [5].

Androgens participate in this process by inducing, in androgen-sensitive body sites, the transformation of thin, non-pigmented vellus hair to coarse, pigmented terminal hair. Terminalization of a hair follicle is a gradual phenomenon but not a reversible one [6].

Besides sebaceous glands, other androgen-sensitive components of skin are hair follicles, sweat glands, epidermis, and dermis. These structures contain enzymes important in converting dehydroepiandrosterone (DHEA), dehydroepiandrosterone sulfate (DHEAS), and androstenedione into the potent androgens dihydrotestosterone (DHT) and testosterone. The DHT and testosterone are the major androgens that interact with the

Address for correspondence: Besa Gacaferri Lumezi MSc, Department of Physiology and Immunology, Medical Faculty, University of Prishtina, Bulevardi i Deshmoreve p.n., 10000 Prishtina, Kosovo, fax: +37744186036, e-mail: besa_gacaferri@yahoo.com Received: 26.04.2014, accepted: 10.09.2014. 
androgen receptors on sebaceous glands with DHT being 5 to 10 times more potent than testosterone $[7,8]$.

\section{Aim}

The aim of pharmacological therapy is to lower serum free androgen levels and/or to block peripheral androgen action. Drugs licensed for the treatment of hyperandrogenism are limited; in some countries, the anti-androgen drug cyproterone acetate may be used for this purpose. In several countries, there are only the topical drug eflornithine or estrogen-progestogen combinations $[9,10]$.

\section{Material and methods}

\section{Patients}

The prospective study included 135 women with hirsutism, aged $14-46$ years. The women sought advice because of hair growth in the androgen sensitive areas, acne, menstrual irregularity, and infertility. Exclusion criteria were premenarchal girls, postmenarchal women, women with corticoid therapy and oral contraceptives, pregnant women or women who after initial evaluation did not come, for blood sampling, at the appointed time.

\section{Clinical evaluation}

A detailed history was taken (marital status, infertility, age of onset of hirsutism, duration of hirsutism, progression of hirsutism, age at menarche, menstrual irregularities, presence of deepening of voice, increased muscle mass, androgen alopecia, galactorrhea, decreased breast size, emotional and mental state, other disorders).

Height, weight and a calculation of body mass index (BMI) was obtained. The normal range of $\mathrm{BMI}$ was taken as $18-24.9 \mathrm{~kg} / \mathrm{m}^{2}$. Women with BMI $25-29.9 \mathrm{~kg} / \mathrm{m}^{2}$ were labeled overweight and those having $30 \mathrm{~kg} / \mathrm{m}^{2}$ or more were diagnosed as obese.

Women were examined for clinical evidence of acne, androgenetic alopecia, acanthosis nigricans, signs of virilization including loss of female body contours, clitoromegaly and atrophy of the breasts. Evaluation of hirsutism was done according to Ferriman-Gallwey score, grading 11 androgen sensitive parts (upper lip, chin, chest, hand, arm, thigh, leg, upper part of back, lower part of back, upper part of abdomen, lower part of abdomen). Grading was done starting with the value 0 (total absence of terminal hair) up to 4 (extended presence of the terminal hair). The value of 8 or above 8 is considered as hirsutism. Acne were evaluated with Hayashi method, based on the number of inflammatory eruptions (mild: $0-5$, moderate: 6-20, severe: $21-50$ and very severe: more than 50 ).

\section{Laboratory assays and other investigations}

Hormones were determined with radioimmunoassay (RIA) and immunoradiometric assay (IRMA) methods.
With RIA we measured hormones: estradiol, progesterone, 17-OH-progesterone, total testosterone, free-testosterone, androstenedione, cortisol, DHEASO4, TSH, T3,T4, and with IRMA method: FSH, LH, prolactin, SHBG and ACTH. Substances used were produced by Immunotech (USA). Blood was taken in the early follicular phase, between the $3^{\text {rd }}$ and $5^{\text {th }}$ day of the menstrual cycle, (8:00 in the morning, and for evening cortisol at 18:00). The serum was separated by centrifugation and stored at $-20^{\circ} \mathrm{C}$. Blood for assessing the progesterone level was taken in the middle of the cycle. Oral glucose tolerance test (OGTT) was done in selected women (positive history for diabetes, polycystic ovarian syndrome (PCOS) and obesity). We measured blood sugar before patients drank the liquid containing $75 \mathrm{~g}$ of glucose. We took blood to measure glucose, insulin and C-peptide every 30 min and 60 min for $3 \mathrm{~h}$.

Polycystic ovarian syndrome was diagnosed by Rotterdam criteria: presence of two of the three elements viz. clinical or biological hyperandrogenism, polycystic ovaries and chronic anovulation. Polycystic ovaries (PCO) were diagnosed on pelvic USG by presence of $\geq 12$ follicles measuring 2-9 $\mathrm{mm}$ in diameter and/or $\geq 10 \mathrm{ml}$ ovarian volume.

Pelvic ultrasound examinations for ovaries and adrenals were carried out in all the women, skull magnetic resonance and abdominal magnetic resonance were carried out in selected women only.

Permission from the ethics committee of the Medical Faculty was obtained.

\section{Statistical analysis}

The collected data were stored in a special database. Statistical processing was done with statistic packet InStat 3. Numerical data were presented as an index of structure, arithmetic mean, standard deviation, minimal value and maximal value. To test the hypothesis between the groups, $t$-test, Mann-Whitney test, a $\chi^{2}$-test, one way ANOVA, Kruskal Wallis test and Fisher exact test were conducted with a significant level of $p<0.05$.

\section{Results}

Evaluation of hirsutism was done according to Ferriman-Gallwey score, grading 11 androgen sensitive areas. The patients who had a normal ovulatory function were diagnosed as having idiopathic hirsutism. The normal ovulatory function was evidenced by normal mid-luteal progesterone and androgen levels. If 17-hydoxyprogesterone levels were raised, the women were diagnosed with congenital adrenal hyperplasia. We diagnosed our patients as having PCOS if two of the following three criteria were present: 1) oligomenorrhea and/or anovulation, 2) hyperandrogenism (clinical or biochemical), and 3) polycystic ovaries shown on ultrasound. 
Table 1. Presence of oily skin in women with hirsutism

\begin{tabular}{|c|c|c|c|c|c|c|c|c|c|c|}
\hline \multirow[t]{3}{*}{ Oily skin } & \multicolumn{8}{|c|}{ Age group [years] } & \multicolumn{2}{|c|}{ Total } \\
\hline & \multicolumn{2}{|c|}{$<20$} & \multicolumn{2}{|c|}{$20-29$} & \multicolumn{2}{|c|}{$30-39$} & \multicolumn{2}{|c|}{$\geq 40$} & \multirow[b]{2}{*}{$n$} & \multirow[b]{2}{*}{$\%$} \\
\hline & $n$ & $\%$ & $n$ & $\%$ & $n$ & $\%$ & $n$ & $\%$ & & \\
\hline Yes & 14 & 66.7 & 40 & 53.3 & 12 & 37.5 & 1 & 14.3 & 67 & 49.6 \\
\hline No & 7 & 33.3 & 35 & 46.7 & 20 & 62.5 & 6 & 85.7 & 68 & 50.4 \\
\hline Total & 21 & 100 & 75 & 100 & 32 & 100 & 7 & 100 & 135 & 100 \\
\hline$\chi^{2}$-test & \multicolumn{8}{|c|}{$\chi^{2}=8.2 ; \mathrm{d} f=3 ; p=0.04$} & & \\
\hline
\end{tabular}

Significant for $p<0.05$

Table 2. Presence of acne in hirsute women

\begin{tabular}{|c|c|c|c|c|c|c|c|c|c|c|}
\hline \multirow{3}{*}{$\begin{array}{l}\text { Presence } \\
\text { of acne }\end{array}$} & \multicolumn{8}{|c|}{ Age group [years] } & \multirow{2}{*}{\multicolumn{2}{|c|}{ Total }} \\
\hline & \multicolumn{2}{|c|}{$<20$} & \multicolumn{2}{|c|}{$20-29$} & \multicolumn{2}{|c|}{$30-39$} & \multicolumn{2}{|c|}{$\geq 40$} & & \\
\hline & $n$ & $\%$ & $n$ & $\%$ & $n$ & $\%$ & $n$ & $\%$ & $n$ & $\%$ \\
\hline Yes & 15 & 71.4 & 40 & 53.3 & 8 & 25 & - & - & 63 & 46.7 \\
\hline No & 6 & 28.6 & 35 & 46.7 & 24 & 75 & 7 & 100 & 72 & 53.3 \\
\hline Total & 21 & 100 & 75 & 100 & 32 & 100 & 7 & 100 & 135 & 100 \\
\hline$\chi^{2}$-test & & & & 18.67; d & $=0$. & & & & & \\
\hline
\end{tabular}

Significant for $p<0.001$

Table 3. Etiology of hirsutism and presence of acne

\begin{tabular}{|c|c|c|c|c|c|c|}
\hline \multirow[t]{3}{*}{ Diagnose } & \multicolumn{4}{|c|}{ Acne } & \multicolumn{2}{|c|}{ Total } \\
\hline & \multicolumn{2}{|c|}{ Yes } & \multicolumn{2}{|c|}{ No } & \multirow[b]{2}{*}{$n$} & \multirow[b]{2}{*}{$\%$} \\
\hline & $n$ & $\%$ & $n$ & $\%$ & & \\
\hline Idiopathic hirsutism & 6 & 20.7 & 23 & 79.3 & 29 & 100.0 \\
\hline Hyperandrogenemia & 23 & 53.5 & 20 & 46.5 & 43 & 100.0 \\
\hline PCOS & 21 & 56.8 & 16 & 43.2 & 37 & 100.0 \\
\hline $\mathrm{NC} C A \mathrm{H}$ & 13 & 50.0 & 13 & 50.0 & 26 & 100.0 \\
\hline Total & 63 & 46.7 & 72 & 53.3 & 135 & 100.0 \\
\hline$\chi^{2}$-test & & $=10$. & -0. & & & \\
\hline
\end{tabular}

Significant for $p<0.05$; $1 \mathrm{H}$-idiopathic hirsutism, HA - hyperandrogenemia, PCOS - polycystic ovary syndrome, NC CAH - non classic congenital adrenal hyperplasia

Hormonal changes in a large number of women with hirsutism causes also the appearance of acne in different parts of the body. In our clinical sample, 63 or $46.7 \%$ have had acne. Sixty-seven or $49.6 \%$ of women with hirsutism had oily skin. We have found that in most cases there is a connection between oily skin and acne $(p<0.001)$. Women with a younger age had oily skin more often than women with older age, so $66.7 \%$ of women under the age of 20 years, $53.3 \%$ of women aged $20-29$ years, $37.5 \%$ of women aged $30-39$ years and $14.3 \%$ of women older than 40 years had oily skin. There were significant differences between age groups ( $p<0.05)$ (Table 1$)$.
From the total of 135 hirsute women, 63 or $46.7 \%$ have had acne. We have found that in most cases there is a connection between acne and age $(p<0.001)$. Younger women had acne more often than older women. $71.4 \%$ of the group of women under the age of $20,53.3 \%$ of women aged $20-29$ and $25.0 \%$ of women aged $30-39$ had acne. There were significant differences between age groups $(p<0.001)$ (Table 2$)$.

Sixty women had mild forms of acne, including: whiteheads, blackheads, papules and pustules. Only 3 women had moderate to severe acne, including: nodules, cysts. Twenty-three or $53.4 \%$ of women with hy- 
Table 4. Localization of acne in hirsute women

\begin{tabular}{|c|c|c|c|c|c|c|c|c|}
\hline \multirow[t]{2}{*}{ Acne } & \multicolumn{2}{|c|}{ Yes } & \multicolumn{2}{|c|}{ No } & \multicolumn{2}{|c|}{ Total } & \multirow[t]{2}{*}{$\chi^{2}$ test } & \multirow[t]{2}{*}{ Value of $p$} \\
\hline & $n$ & $\%$ & $n$ & $\%$ & $n$ & $\%$ & & \\
\hline Face & 39 & 29.1 & 96 & 70.9 & 135 & 100 & 24.1 & $<0.001$ \\
\hline Forehead & 25 & 18.5 & 110 & 81.5 & 135 & 100 & 53.5 & $<0.001$ \\
\hline Chin & 36 & 26.7 & 99 & 73.3 & 135 & 100 & 29.4 & $<0.001$ \\
\hline Upper lip & 5 & 3.7 & 130 & 96.3 & 135 & 100 & 115.7 & $<0.001$ \\
\hline Chest & 8 & 5.9 & 127 & 94.1 & 135 & 100 & 104.9 & $<0.001$ \\
\hline Back & 15 & 11.1 & 120 & 88.9 & 135 & 100 & 81.7 & $<0.001$ \\
\hline Other parts of the body & 2 & 1.5 & 133 & 98.5 & 135 & 100 & 186.1 & $<0.001$ \\
\hline
\end{tabular}

Table 5. The BMI in hirsute women with acne

\begin{tabular}{|c|c|c|c|c|c|c|c|c|c|c|}
\hline \multirow[t]{2}{*}{ Diagnosis } & \multicolumn{2}{|c|}{ Underweight } & \multicolumn{2}{|c|}{ Normal } & \multicolumn{2}{|c|}{ Overweight } & \multicolumn{2}{|c|}{ Obese } & \multicolumn{2}{|c|}{ Total } \\
\hline & $n$ & $\%$ & $n$ & $\%$ & $n$ & $\%$ & $n$ & $\%$ & $n$ & $\%$ \\
\hline $\mathrm{IH}$ & 0 & 0.0 & 5 & 83.3 & 1 & 16.7 & 0 & 0.0 & 6 & 100.0 \\
\hline HA & 4 & 17.4 & 13 & 56.5 & 3 & 13.0 & 3 & 13.0 & 23 & 100.0 \\
\hline PCOS & 3 & 14.3 & 11 & 52.4 & 4 & 19.0 & 3 & 14.3 & 21 & 100.0 \\
\hline NC CAH & 1 & 7.7 & 8 & 61.5 & 4 & 30.8 & 0 & 0.0 & 13 & 100.0 \\
\hline Total & 8 & 12.7 & 37 & 58.7 & 12 & 19.0 & 6 & 9.5 & 63 & 100.0 \\
\hline$\chi^{2}$-test & & & & $=6.3 ;$ & $p=$ & & & & & \\
\hline
\end{tabular}

Not significant $p>0.05$. IH - idiopathic hirsutism, HA - hyperandrogenemia, PCOS - polycystic ovary syndrome, NC CAH - non classic congenital adrenal hyperplasia

perandrogenemia had acne, 21 or $56.7 \%$ of women with PCOS had acne, 13 or $50.0 \%$ of women with non classic congenital adrenal hyperplasia (NC CAH) had acne and 6 or $20.6 \%$ of women with idiopathic hirsutism had acne. There were significant differences between groups with hirsutism and acne and the group with hirsutism without acne, because women with PCOS more often have acne $(p<0.05)$ (Table 3).

In our clinical sample, 39 or $29.1 \%$ of women had acne on the face, 25 or $18.5 \%$ on the forehead, 36 or $26.7 \%$ on the chin, 5 or $3.7 \%$ on the upper lip, 8 or $5.9 \%$ on the chest, 15 or $11.1 \%$ on the back and 2 or $1.5 \%$ on other parts of the body. The most common localization of acne was the face and chin and the least was in other parts of body. There were significant differences between sites of localization of acne $(p<0.001)$ (Table 4).

In the group of 63 women diagnosed with hirsutism and who had acne, testosterone was elevated in 49 women or $79 \%$, free testosterone was increased in 13 women or $20.6 \%$, androstenedione in 44 women or $69.8 \%$, DHEAS was increased in 19 women or $30.1 \%$, 17-OH-progesterone in 43 women or $68.2 \%$ and sex hormone-binding globulin (SHBG) was decreased in 21 women or $33.3 \%$. Six women had idiopathic hirsutism and acne.
Table 6. Improvement of acne after using therapy

\begin{tabular}{|c|c|c|c|}
\hline Improvement & $N$ & $\%$ & $\chi^{2}$-test \\
\hline No improvement & 29 & 21.5 & \multirow{3}{*}{$\begin{array}{c}\chi^{2}=10.7 \\
\mathrm{~d} f=2 ; \\
p=0.01\end{array}$} \\
\hline Slight improvement & 9 & 6.7 & \\
\hline Significant improvement & 25 & 18.5 & \\
\hline No acne & 72 & 53.3 & \\
\hline Total & 135 & 100.0 & \\
\hline
\end{tabular}

In this group 12 women or $19 \%$ were overweight, of whom 3 (4.7\%) with PCOS, 4 (6.3\%) with NC CAH, and $3(4.7 \%)$ women with hyperandrogenemia. In the same group of 63 women, 6 or $9.5 \%$ were obese, from whom 3 or $4.7 \%$ had hyperandrogenemia and 3 women or $4.7 \%$ had PCOS. There were no significant differences in BMI between etiology groups ( $p>0.05$ ) (Table 5).

Forty-four of 63 women who received only oral contraceptives or combined with other medication showed improvement, 9 of them slight improvement and 25 significant improvement. Nineteen women who did not use any therapy had no improvement in acne but they had improvement in hirsutism because of laser therapy. There were no significant differences between groups $(p<0.01)$ (Table 6). 


\section{Discussion}

Acne vulgaris is a multifactorial disease that is hormonally mediated and genetically controlled [11]. It is the most common dermatosis found in patients aged between 11 and 30. However, there are cases when disease is still present until 30 or even 40 years of age. Ninety-five percent of changes are localized on the face and on the upper part of the trunk, rarely on other body parts and this disease frequently constitutes a serious psychological problem for the patient because of its localization and chronic character [12].

In our study from the total of 135 hirsute women, 63 or $46.7 \%$ have had acne. Sharma in his study found that $64 \%$ of hirsute women had acne [13], which result is slightly higher than ours. When analyzed by age factor, $71.4 \%$ of the group of women under the age of 20 , and $25.0 \%$ of women aged 30-39 had acne. The most common localization of acne in hirsute women was the face and the least was in other parts of body

Our study showed that $56.7 \%$ of women with PCOS had acne. The prevalence of acne in women with PCOS has been estimated to be 10-34\%. However, in post-pubertal and adolescent PCOS women it is unclear whether acne arises secondary to androgen excess or if it occurs as a result of normal puberty [14].

In this study, $50.0 \%$ of women with NC CAH had acne. Moran et al. reviewing the distribution of symptoms in 220 women from 11 centers, found that acne were present in 33\% of women with NC CAH [15].

Most adult women with acne have a normal circulating level of androgens and require no work-up. In our study only 6 or $20.6 \%$ of women with idiopathic hirsutism had acne.

Androgens (total testosterone, androstenedione, dehydroepiandrosterone sulfate) are involved in the development of acne [16]. In this study we found a high prevalence of androgen excess among hirsute women with acne: total testosterone was increased in 49 women or $79 \%$, free testosterone in 13 or $20.6 \%$, androstenedione in 44 or $69.8 \%$, DHEAS in 19 or $30.1 \%, 17-\mathrm{OH}$-progesterone in 43 or $68.2 \%$ and SHBG was decreased in 21 or $33.3 \%$. Karrer-Voegeli, Rahman, Slayden reported similar results with ours [16-18]. Zaenglein et al. reported normal androgens [19].

The BMI is defined as weight in kilograms divided by height in meters squared. The BMI distribution among groups is not statistically significant in our study. We found that 81 (60.0\%) of all hirsute women had normal weight, only 13 (9.6\%) were obese. In the group of women with hirsutism and acne, 6 (9.5\%) were obese, among whom 3 (4.7\%) had hyperandrogenemia and $3(4.7 \%)$ women had PCOS. The prevalence of obesity was lower than in other studies. Bazarganipour et al. and Saxena et al. reported a higher prevalence of obesity than in our study [20, 21].
Every oral contraceptive is not effective in acne treatment [22]. Norgestimate ethinyl estradiol, norethindrone ethinyl estradiol and drospirenone ethinyl estradiol have been approved by the FDA in acne treatment [23].

In our sample of 63 women with hirsutism and acne, 44 women (69\%) have received oral contraceptives for a year. Twenty women (31\%) received only oral contraceptives and 24 women (38\%) received oral contraceptives combined with other medications (Dexason, Metformin, Spironolactone, Bromergon), the other women (19 or 31\%) refused therapy. The first improvements in hirsutism and acne were seen after 6 months. After a year of therapy, 34 women (53.9\%) showed improvement in acne and hirsutism. Improvement has been more pronounced in women who used combined therapy. Vloten in his study reported similar results [24]. Koo et al. compared the efficacy of antibiotics and oral contraceptives in managing acne. Results suggest that although antibiotics may be superior at 3 months, oral contraceptives are equivalent to antibiotics at 6 months in reducing acne lesions and, thus, may be better firstline alternative to systemic antibiotics for long-term acne management in women [25].

\section{Conclusions}

In this study we found a high prevalence of acne in hirsute women. Prevalence of acne was higher in PCOS. Acne were more localized on the face and associated with elevated androgens. The BMI was not statistically significant among all groups. Since these women have associated endocrine changes it is important to correct them with hormonal therapy.

\section{Acknowledgments}

We are thankful to the medical students who participated in the study.

\section{References}

1. Ballanger F, Baudry P, N'Guyen JM, et al. Heredity: a prognostic factor for acne. Dermatology 2006; 212: 145-9.

2. Voegeli SK, Rey F, Reymond MJ, et al. Androgen dependence of hirsutism, acne and alopecia in women. Medicine 2009; 88: $32-45$

3. Cibula D, Hill M, Vohradnikova O, et al. The role of androgens in determining acne severity in adult women. Br J Dermatol 2000; 143: 399-404.

4. Azziz R, Sanchez LA, Knochenhauer ES, et al. Androgen excess in women: experience with over 1000 consecutive patients. J Clin Endocrinol Metab 2004; 89: 453-62.

5. Seirafi H, Farnaghi F, Farhani AV, et al. Assessment of androgens in women with adult-onset acne. Int I Dermatol 2007; 46: 1188-91.

6. Azziz R, Carmina E, Sawaya ME. Idiopathic hirsutism. Endocr Rev 2000; 21: 347-3. 
7. Clarke S, Nelson A, George R, et al. Pharmacologic modulation of sebaceous gland activity: mechanisms and clinical applications. Dermatol Clin 2007; 25: 137-46.

8. Thiboutot D, Chen W. Update and future of hormonal therapy in acne. Dermatology 2003; 206: 57-67.

9. Guido M, Romualdi D, Giuliani M, et al. Drospirenone for the treatment of hirsute women with polycystic ovary syndrome: a clinical, endocrinological, metabolic pilot study. J Clin Endocrinol Metabol 2004; 89: 2817-23.

10. Palep-Singh M, Mook K, Barth J, Balen A. An observational study of Yasmin in the management of women with polycystic ovary syndrome. J Fam Plann Reprod Health Care 2004; 30: 163-5.

11. Gold MH. Evaluating cosmetic outcomes and patient compliance when selecting therapies for acne vulgaris. Cosmet Dermatol 2011; 24: 432-6.

12. Bergler-Czop B, Brzezińska-Wcisło L. Pro-inflammatory cytokines in patients with various kinds of acne treated with isotretinoin. Postep Derm Alergol 2014; 31: 21-8.

13. Sharma NL, Mahajan VK. Hirsutism: clinico-investigative profile of 50 Indian patients. Indian J Dermatol 2008; 53: 111-4.

14. Chuan SS, Chang RJ. Polycystic ovary syndrome and acne. Skin Therapy Letter 2010; 15: 1-4.

15. Moran C, Azziz R, Carmina E, et al. 21-hydroxylase-deficient nonclassic adrenal hyperplasia is a progressive disorder: a multicenter study. Am J Obstet Gynecol 2000; 183: 1468-74.

16. Karrer-Voegeli S, Rey F, Reymond MJ, et al. Androgen dependence of hirsutism, acne, and alopecia in women: retrospective analysis of 228 patients investigated for hyperandrogenism. Medicine (Baltimore) 2009; 88: 32-45.

17. Slayden SM, Moran C, Sams JWM, et al. Hyperandrogenemia in patients presenting with acne. Fertil Steril 2001; 75 : 889-92.

18. Rahman M, Sikder AU, Rashid MM, et al. Association of serum testosterone with acne vulgaris in women. BSMMU J 2012; 5: 1-5.

19. Zaenglein AL, Garber EM, Thiboutot DM, Strauss JS. Acne vulgaris and acneiform eruptions. In: Fitzpatrick's dermatology in general medicine. 7th ed. McGraw-Hill Publisher, San Fransisco, USA 2008; 690-6.

20. Bazarganipour F, Ziae S, Montazeri A, et al. Health-related quality of life and its relationship with clinical symptoms among Iranian patients with polycystic ovarian syndrome. Iran J Reprod Med 2013; 11: 371-8.

21. Saxena P, Prakash A, Nigam A, Mishra A. Polycystic ovary syndrome: is obesity a sine qua non? A clinical, hormonal, and metabolic assessment in relation to body mass index. Indian J Endocrinol Metabol 2012; 16: 996-9.

22. Tatasiewicz K, Oldakowska A, Szczerkowska-Dobosz A. Evaluation of knowledge about acne vulgaris among a selected population of adolescents of Tricity schools. Postep Derm Alergol 2012; 29: 417-20.

23. Salvaggio HL, Zaenglein AL. Examining the use of oral contraceptives in the management of acne. Int I Womens Health 2010; 2: 69-76.

24. Van Vloten WA, van Haselen CW, van Zuuren EJ, et al. The effect of 2 combined oral contraceptives containing either drospirenone or cyproterone acetate on acne and seborrhea. Cutis 2002; 69: 2-15.

25. Koo EB, Petersen TD, Kimball AB. Meta-analysis comparing efficacy of antibiotics versus oral contraceptives in acne vulgaris. J Am Acad Dermatol 2014; 71: 450-9. 